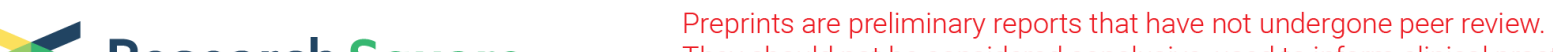 Research Square They should not be considered conclusive, used to inform clinical practice, or referenced by the media as validated information.
}

\section{The ECM and CAFs interact to differentially regulate PDAC parenchymal (CPC) and CSC phenotypes to determine pancreatic ductal adenocarcinoma (PDAC) progression}

Stefania Cannone

University of Bari

Maria Rafaella Greco

University of Bari

Hélène Guizouarn

University of Nice

Olivier Soriani

University of Nice

Richard Tomasini

Institut Paoli-Calmettes, CNRS, UMR7258

Valeria Casavola

Universita degli Studi di Bari Aldo Moro

Katrine Zeeberg

Universita degli Studi di Bari Aldo Moro

Stephan Joel Reshkin ( $\nabla$ stephanjoel.reshkin@uniba.it )

University of Bari https://orcid.org/0000-0001-9757-5908

\section{Rosa Angela Cardone}

Universita degli Studi di Bari Aldo Moro

Research article

Keywords: Desmoplastic Reaction, Cancer stem cells, Cancer Associated Fibroblasts, Pancreatic Adenocarcinoma, Vasculogenic Mimicry, 3D organotypic cultures

Posted Date: August 20th, 2019

DOI: https://doi.org/10.21203/rs.2.13193/v1

License: (9) (1) This work is licensed under a Creative Commons Attribution 4.0 International License. Read Full License 


\section{Abstract}

Summary CAFs and acellular stromal ECM components interact to drive metastatic progression by stimulating the hallmark behaviors of each tumor cell type that contribute to metastasis: invasion in the CPCs and growth and angiogenesis in the CSCs.

\section{Background}

Pancreatic Ductal Adenocarcinoma (PDAC) is one of the most lethal cancers having a five-year survival rate of less than $8 \%$ [1], [2] and will become the second cause of cancer deaths in the coming years [3], [4]. One of the aggressive characteristics of PDAC is their highly reactive and tumor-supportive stromal microenvironment named desmoplasia [5]. Desmoplasia is a dense extracellular matrix (ECM), which makes up to $90 \%$ of PDAC tissue [6, 7] [8, 9], [10] and is characterized by increasing collagen type I levels as the tumor progresses and which contains the cancer cells and their accessory cells, including cancer associated fibroblasts (CAFs) [11], [12]. Collagen I makes up to $80 \%$ of the tumor space in PDAC and is associated with a worsened outcome $[13,14]$ and stimulates malignant cell properties to promote tumor growth, early metastasis and chemo-radiation resistance [15] [14] [9] [16]. Cancer stem cells (CSCs), which drive tumor heterogeneity and influence tumorigenesis, metastasis and drug resistance through their self-renewal and multi-lineage differentiation capabilities (stemness) (for review see [17]).

Recent studies have demonstrated that the stromal ECM composition "per se" produces important cues that guide the expression of different PDAC phenotypes in both parenchymal cancer cells (CPCs) [18],[5] and CSCs [5]. In particular, ECM composition differently regulates growth, morphology, invasive, angiogenic capacities and secretome profiles in PDAC CPCs and their derived CSCs [5]. Importantly, in that study only the CSCs secreted factors known to activate and maintain CAFs19], [20]. This suggests that the described dual "symbiotic", mutual support interaction between tumor cells and CAFs is maintained primarily through the CSC population through the existence of a tighter relationship between CSCs and CAFs than between the CPCs and CAFs. However, this has yet to be demonstrated.

Once activated, CAFs enhance the development, progression and invasion of PDAC through their extensive crosstalk with the tumor, resulting in reciprocal stimulation and therapy resistance [21], [22]. Recent data in PDAC has shown that CAF cells, via their secretome, can increase parenchymal tumor cell (CPC) invasion [23], [24], [25], [26], reduce their growth [23], [27] and modify their epigenetic and metabolic phenotypes [24,27]. Only one study [27] measured the effect of the combination of CAF secretome and high levels of ECM collagen I on parenchymal PDAC cell lines (CPCs) but did not determine the individual roles of collagen I and the CAF (Conditioned Medium). Importantly, those in vitro experiments were not performed on CSCs and, therefore, the effect of CAFs on CSC behavior in PDAC is completely unknown. Nor is the contribution of the ECM in the modulation of the CAF-driven determination of CPC and CSC phenotypic plasticity and behaviour known. 
Given the complex interactions between cancer cells and CAFs, more work is needed to investigate the contributions of CAFs in enabling or maintaining hallmark behaviors in CSCs. Here, we analyzed the role of ECM collagen I in modulating the effect of CAF-derived signals with conditioned medium from primary cultured CAFs derived from patients with PDAC on the parenchymal (CPC) and CSC populations in a previously described three-dimensional (3D) organotypic model of PDAC [5] [18] mimicking the ECM of early (low collagen I levels) and late (high collagen I levels) stage PDAC tumors.

These data reveal that the cellular (CAFs) and acellular (ECM) stromal components interact to differently modulate the hallmark tumor behaviors in the CSCs and CPCs. In particular, the CAFs reduce the growth of CPCs while favoring the growth of CSCs, which would trigger a positive feedback mechanism to stimulate CSC growth and make for a more malignant, persistent and immortal tumor.

\section{Methods}

\section{Cell lines}

The human pancreatic adenocarcinoma parenchymal (CPC) cell line, Panc1, and Panc1 CSCs, generated as previously described [28], were grown and maintained in standard conditions as previously described [5]. Panc1 cells express the mutated PDAC driver genes KRAS, CDKN2A, MAP2K4, and TP53 [29], [30].

\section{Cancer Associated Fibroblasts (CAFs)}

The experimental procedure relating to the use of patient-derived pancreatic tumor pieces was performed after approval from the South Mediterranean Personal Protection Committee, under the reference 2011A01439-32. CAFs isolation and culture were processed as previously described [31]. Briefly, pancreatic tissues obtained during pancreatic surgery from patients with resectable pancreatic adenocarcinoma were cut into small pieces of $1 \mathrm{~mm}^{3}$ using a razor blade. The dissociation of these tissue pieces and the culture to isolate CAFs were performed as in [31] and primary CAF were verified by positive a-SMA staining and negative KRT19 immunofluorescence staining.

To collect conditioned media (CM), $1.5 \times 10^{5} \mathrm{CAF}$ cells/well were seeded in 24-well cell culture plates and medium changed every 3 days. When the monolayer reached approximately $80 \%$ confluence, they were incubated with $1 \mathrm{ml}$ of medium with $1 \%$ FBS lacking growth factors and antibiotics for 30 hours. The CM were collected, centrifuged, protein concentration assayed with the Bradford protein assay reagent (Pierce, Milan, Italy) using bovine serum albumin as a standard and stored in liquid nitrogen.

\section{D culture models}

The $90 \%$ Matrigel-10\% Collagen I and 20\% Matrigel - $80 \%$ Collagen I ECM mixtures were prepared as previously described [5]. In all cases, $100 \mu \mathrm{l}$ of the mixture were plated in 96 -well cell culture plates, which 
were then incubated at $37^{\circ} \mathrm{C}$ with $5 \% \mathrm{CO}_{2}$ for 1 hour to allow the mixture to create a thin gel on the bottom of the wells. $1.5 \times 10^{4}$ cells/well were seeded on the top of the matrix and cultured as described above.

\section{Indirect co-culture}

To condition cells, both CPCs and CSCs were grown for 1 day on $90 \% \mathrm{M}: 10 \% \mathrm{C}$ or $20 \% \mathrm{M}: 80 \% \mathrm{C}$ in their corresponding complete culture media and for the subsequent 5 days in either $100 \%$ CM collected from the primary patient CAFs or in the CM diluted at $50 \%$ with the same complete culture media used to grow the cells. A change of medium was conducted midweek. To analyze if the CAF CMs could change the growth and/or the growth phenotype (morphology) of the cells, growth was assessed by the Resazurin cell viability assay and morphology was examined microscopically.

\section{Growth measurements}

Curves of CPCs and CSCs were calculated from Resazurin (Immunological Sciences, Rome, Italy) reduction assays for cell growth as previously described [32]. Standard curves obtained by fluorescence readings of Resazurin on serial dilutions of both CPCs and CSCs were used to calculate cell number.

\section{Invadopodia proteolytic activity}

Experiments to measure invadopodia focal ECM proteolysis were conducted in cells seeded onto a ECM layer in which quenched BODIPY linked to BSA (DQ-Green-BSA, Thermo Fisher Scientific) was mixed at a final concentration of $30 \mu \mathrm{g} / \mathrm{ml}$ as previously described [5].

\section{Vascular network analysis}

CSCs and CPCs were grown on the $90 \%$ Matrigel:10\%Collagen I (90\%M:10\%C) ECM mixture. After 5 days in these growth conditions, vascular channel networks were photographed using the TE200 microscope (Nikon USA, Garden City, NY, USA) and analysed as previously described [5]. To study paracrine CAF regulation of vascular parameters, CSCs or parenchymal cells were cultured as above and after $24 \mathrm{hrs}$, to permit their adherence, the cultures were incubated with CAF CM as described above for indirect coculture.

\section{Statistical analysis}

GraphPad Prism 5 (GraphPad Software) to perform a two-tailed Student's $t$ test assuming unequal variances to compare the effects of CAF CM on Panc1 CPCs and CSCs and to determine whether the 
differences between two groups were statistically significant. P-values $<0.05,0.01$, or 0.001 are indicated as $*, * *$ or $* \star *$, respectively when compared to each control for each matrix and ${ }^{t, t+}$ or ${ }^{t+t}$ compared to the same CM treatment on $90 \%$ Matrigel: $10 \%$ Collagen I.

\section{Results}

Given the above described crosstalk between CAFs and cancer cells and the reported role of the ECM composition in regulating the phenotypes of both PDAC CPCs [5] [18] and CSCs [5], we evaluated the role of the ECM composition on the effect of two concentrations of Conditioned Medium (CM) derived from CAFs isolated from a PDAC patient on the growth, invasive capacity and vascular morphology of each cell type. The experiments were performed with the cells cultured on either $90 \%$ Matrigel: $10 \%$ Collagen I or $20 \%$ Matrigel: $80 \%$ Collagen I since these combinations very well mimic the ECM of early and late stages of PDAC progression, respectively (see Materials and Methods and protocol scheme in Supplemental Figure 1).

\section{CAF CM reduces CPC growth and increases CSC growth on all of the substrates.}

When CPCs or CSCs were incubated with two concentrations of CAF CM on the two substrates, we observed that CAF CM reduces growth of the CPCs and increases growth of the CSC in a progressive, dose-dependent manner that was independent of ECM composition with a similar behavior on both early $(90 \% \mathrm{M}: 10 \% \mathrm{C})$ and late $(20 \% \mathrm{M}: 80 \% \mathrm{C}) \mathrm{ECM}$ compositions (Figure 1). This would favor the expansion of the CSCs over the CPCs, in line with the report that only the CSC population secreted factors known to activate CAFs [5] and supports the hypothesis of a mutual "symbiotic" support between these two cell types as in other tumor types [33] [34]. Further, this would support the reports that CAFs drive an increase in general 'stemness' of the tumor in various other tumor types and would suggest that, at least in PDAC, this occurs by a net favoring of CSC growth over CPC growth.

\section{Effect of primary CAF CM on CPC and CSC invadopodia activity}

We have previously observed that the PDAC CPCs have higher levels of invadopodia-driven invasion than do the CSCs and that this phenomenon is greater on Matrigel rich ECMs than on collagen I ECMs [5]. We, therefore, next analyzed the role of the ECM composition in modulating the CAF CM-dependent regulation of the invasive phenotype of the CPCs and CSCs, by measuring their invadopodia-mediated ECM proteolytic activity defined by their Digestion Index [35] (Figure 2B). As previously reported [5], we found that (i) the control CPCs had a much higher ability to form functional invadopodia and digest the two types of ECM compared to control CSCs and (ii) both cell types had a higher Digestion Index when cultured on $90 \% \mathrm{M}: 10 \% \mathrm{C}$ with respect to $20 \% \mathrm{M}: 80 \% \mathrm{C}$. 
The effects of the CAF CM on the formation and activity of invadopodia were very complex. On $90 \% \mathrm{M}: 10 \% \mathrm{C}$, treatment with $50 \% \mathrm{CM}$ produced a small but not significant increase in the CPC invadopodia activity while $100 \% \mathrm{CM}$ stimulated CPC invadopodial activity 2.5 -fold. CAF CM had no significant effect on CSC invadopodia activity at either concentration. Interestingly, when cultured on 20\%M:80\%C neither cell line responded significantly to CAF CM at either concentration.

\section{Effect of primary CAF CM on CPC and CSC vasculogenic mimicry}

We have previously reported that vascular-like structures correspondent with in vivo Vasculogenic Mimicry (VM) were formed by the CSCs on $100 \%$ Matrigel and were reduced as Collagen I levels increased and completely disrupted as Collagen I levels surpassed $30 \%$ of the ECM composition [5]. We asked whether the CAF CM-driven stimulation of CSC growth observed in Figure 1 would also result in an increase of VM organization in the CSCs and, perhaps, in a slight increase in VM in the CPC population. Indeed, when CSCs or CPCs were incubated with the two concentrations of CAF CM on the $90 \% \mathrm{M}: 10 \% \mathrm{C}$ substrate, we observed that CAF CM increases both the mean number of lacunae per well and number of capillary connections to nodes per field of the CSCs in a progressive, dose-dependent manner that resulted in the formation of very tight, organized vascular structures. Interestingly, CAF-CM somewhat stimulated a capillary-like phenotype also in the CPCs but only at the higher concentration of CAF CM, suggesting that it may induce the epithelial-mesenchymal transition of CPCs.

\section{Discussion}

Utilizing a 3D organotypic culture model representing the ECM changes observed during in vivo PDAC progression from a laminin-rich to a collagen I-rich environment [36], we have previously found that the ECM composition drove the very early development of metastatic behaviors of both the parenchymal tumor cell (CPC) and CSC populations which is another of the important PDAC characteristics [14], [37], [38]. Indeed, when growing in an early tumor ECM modeled by the Matrigel rich ECM (containing laminin, collagen IV and entactin), the CSCs secreted high levels of pro-angiogenic/growth factors, which activate their transdifferentiation into an endothelial-like VM network via a VEGF/VEGFR-2 mediated cascade. At the same time, when on Matrigel the more differentiated CPC population has a high invadopodia-driven invasive capacity that is stimulated by EGF [39], which is highly secreted by the CSCs. This concerted and reciprocal activation of these two malignant phenotypes, i.e. (1) the high rate of local invasion executed by CPCs into the (2) aberrant vascular network created by the CSC-derived vascular signals suggested a symbiotic relationship between the CPCs and the CSCs underlies the initiation and maintenance of early PDAC infiltration and metastasis. In this way, parenchymal CPC cells and CSCs interact to contribute to the vascular [40] and invasive phenotype of the early stage tumor.

However, tumor growth and phenotypes are influenced not only by their own gene expression-related and ECM-related factors, but also via the interaction of the tumor cells with the accessory cells found in the 
tumor stroma. In PDAC, the major stroma cell driving progression is considered to be the Cancer Associated Fibroblasts (CAFs) and due to the sparse distribution of tumor cells the interaction between the different cell types occurs principally via the soluble factors secreted by both tumor cells and CAFs [23] [24] [25] [26]. While in other tumor types, the interaction of CSCs with CAFs has been documented, in PDAC the role of CAFs in determining and/or modulating the balance between the parenchymal and CSC tumor populations is still unknown.

Here, utilizing our organotypic cultures mimicking the changes in ECM composition with PDAC progression, we characterized the role of CAFs on both CPC and CSC phenotypes when cultured on ECM compositions known to drive specific growth and phenotype patterns in the two tumor cell types. We find that when growing on an early tumor ECM (modeled by $90 \% \mathrm{M}: 10 \% \mathrm{C}$ ), the CAFs increased both CSC growth (Fig. 1A) and their assembly into a VM network (Fig. 3). Together, this increases their dedicated programing towards the preparation of a vascular niche and eventual transdifferentiation into an endothelial-like network [5]. At the same time, when on Matrigel the CAFs further reduced the growth rate of the more differentiated CPC cell population (Fig. 1B) but greatly increased their already high invadopodia-driven invasive capacity (Fig. 2). This concerted over-activation of these two malignant phenotypes by the CAFs, i.e. (1) the high rate of CPC local invasion into the (2) CSC-derived vasculogenic network suggest that the CAFs specifically activate the previously described symbiotic relationship between the parenchymal CPC cells and the CSCs that underlies early PDAC infiltration and metastasis [5].

It has been previous reported [5] that CSCs develop their vascular phenotype on Matrigel via the coordinated interaction of two factors: (i) the intrinsic over-expression of genes for endothelial factors and vascular receptors and (ii) the high secretion of VEGF from both the CSCs and CPCs. Based on the findings reported here, this program is further stimulated by a third factor, the secretion by CAFs of various pro-angiogenic/growth factors. Altogether, these factors support their high growth rate necessary to form the vascular network. This would both mobilize CSCs (together with endothelial cells, pericytes etc) into a vascular niche and further stimulate the CSCs endothelial-like differentiation program, in-orderto participate in vasculogenic mimicry, and their angiogenic secretome to stimulate endothelial angiogenesis [41]. This enhanced ability of the CAFs to stimulate the previously described CSC growth/pro-angiogenic program [5], especially in response to an early stage tumor microenvironment (eg. growth on Matrigel), is in line with in vivo experiments in which (a) subcutaneous tumors originating from CSCs gave rise to amore abundant vascular network composed of larger vessels than the tumors originating from CPCs, (b) the tumors of the CSC-injected mice had increased $\mathrm{Ki}-67$ staining for mitotic index [5] and (c) grow faster than those in mice injected with CPCs [28].

\section{Conclusions}

In conclusion, our study sheds light on the role of the CAFs in driving the very early metastatic development, one of the most representative PDAC hallmarks [38]. We find that the CAFs and acellular stromal components interact to modulate the hallmark tumor behaviors of the CPC and CSC cell types 
and drive metastatic progression by stimulating the hallmarks of each tumor cell type that contribute to metastasis: invasion in the CPCs and growth \& angiogenesis in the CSCs (Figure 4). Altogether, these findings propose a scenario in which the ECM composition and the cellular secretomes of the two tumor cell types and the CAFs cooperate to jointly regulate both growth and morphology of the CPC and CSC cell lines and, by modulating the highly dynamic interactions between them, establishes a continuum between tumor initiation and progression in primary PDAC tumors. This suggests that also in PDAC, such as in other tumor types, CAFs drive progression by favoring the growth and vascular plasticity of the CSC population.

\section{Abbreviations}

PDAC: Pancreatic Ductual AdenoCarcinoma; CAF: Cancer Associated Fibroblasts; ECM: ExtraCellular Matrix; CPC: Cancer Parenchymal Cells; CSC: Cancer Stem Cells; 3D: three dimensional culture; VM: Vasculogenic Mimicry; M: Matrigel; C: Collagen I; CM: Conditioned Medium

\section{Declarations}

\section{Ethics approval and consent to participate}

The experimental procedure relating to the use of patient-derived pancreatic tumor pieces was performed after approval from the South Mediterranean Personal Protection Committee, under the reference 2011A01439-32.

\section{Consent for publication}

Not applicable

\section{Availability of data and material:}

The datasets used and/or analysed during the current study are available from the corresponding author on reasonable request.

\section{Competing interests:}

The authors declare that they have no competing interests

\section{Funding}


This work was supported by Fondation de France, (2013-00038330; 2015-00059283), Fondation ARC (PJA 20161204740), Ligue contre le Cancer (GB/MA/IQ-10607) (OS, HG). KZ was a fellow of the Marie Curie Initial Training Network IonTraC (FP7-PEOPLE-2011-ITN Grant Agreement No. 289648) to SJR. The SJR laboratory is part of the Italian network "Istituto Nazionale Biostrutture e Biosistemi" (INBB), and the project BioBoP of the Region Puglia. The funding bodies had no involvement in study design; in the collection, analysis and interpretation of data; in the writing of the report; and in the decision to submit the article for publication.

\section{Authors' contributions}

SJR, MRG, RAC, and KZ organized the project and designed the experiments. SJR, RAC and VC wrote the manuscript. MRG, SC, conducted the experiments, performed the image analyses and statistical evaluation. RT provided the CAF population from patient tissue and OS and HG provided the conditioned medium from the CAF cell cultures.

All authors have read and approved the manuscript

\section{Acknowledgements}

Not applicable

\section{References}

[1] Hong SM, Park JY, Hruban RH, Goggins M. Molecular signatures of pancreatic cancer. Arch Pathol Lab Med. 2011;135:716-27.

[2] Di Marco M, Grassi E, Durante S, Vecchiarelli S, Palloni A, Macchini M, et al. State of the art biological therapies in pancreatic cancer. World J Gastrointest Oncol. 2016;8:55-66.

[3] Siegel RL, Miller KD, Jemal A. Cancer Statistics, 2017. CA: a cancer journal for clinicians. 2017;67:730 .

[4] Schober M, Jesenofsky R, Faissner R, Weidenauer C, Hagmann W, Michl P, et al. Desmoplasia and chemoresistance in pancreatic cancer. Cancers (Basel). 2014;6:2137-54.

[5] Biondani G, Zeeberg K, Greco MR, Cannone S, Dando I, Pozza ED, et al. Extracellular Matrix composition modulates PDAC parenchymal and stem cell plasticity and behavior through the secretome. FEBS J. 2018.

[6] Neesse A, Michl P, Frese KK, Feig C, Cook N, Jacobetz MA, et al. Stromal biology and therapy in pancreatic cancer. Gut. 2011;60:861-8. 
[7] Erkan M, Hausmann S, Michalski CW, Fingerle AA, Dobritz M, Kleeff J, et al. The role of stroma in pancreatic cancer: diagnostic and therapeutic implications. Nat Rev Gastroenterol Hepatol. 2012;9:45467.

[8] Liang C, Shi S, Meng Q, Liang D, Ji S, Zhang B, et al. Complex roles of the stroma in the intrinsic resistance to gemcitabine in pancreatic cancer: where we are and where we are going. Experimental \& molecular medicine. 2017;49:e406.

[9] Liang C, Shi S, Meng Q, Liang D, Ji S, Zhang B, et al. Do anti-stroma therapies improve extrinsic resistance to increase the efficacy of gemcitabine in pancreatic cancer? Cellular and molecular life sciences: CMLS. 2018;75:1001-12.

[10] Veenstra VL, Garcia-Garijo A, van Laarhoven HW, Bijlsma MF. Extracellular Influences: Molecular Subclasses and the Microenvironment in Pancreatic Cancer. Cancers (Basel). 2018;10.

[11] Ying H, Dey P, Yao W, Kimmelman AC, Draetta GF, Maitra A, et al. Genetics and biology of pancreatic ductal adenocarcinoma. Genes Dev. 2016;30:355-85.

[12] Dalla Pozza E, Forciniti S, Palmieri M, Dando I. Secreted molecules inducing epithelial-tomesenchymal transition in cancer development. Semin Cell Dev Biol. 2017.

[13] Whatcott CJ, Diep CH, Jiang P, Watanabe A, LoBello J, Sima C, et al. Desmoplasia in Primary Tumors and Metastatic Lesions of Pancreatic Cancer. Clin Cancer Res. 2015;21:3561-8.

[14] Shields MA, Dangi-Garimella S, Redig AJ, Munshi HG. Biochemical role of the collagen-rich tumour microenvironment in pancreatic cancer progression. Biochem J. 2012;441:541-52.

[15] Koenig A, Mueller C, Hasel C, Adler G, Menke A. Collagen type I induces disruption of E-cadherinmediated cell-cell contacts and promotes proliferation of pancreatic carcinoma cells. Cancer Res. 2006;66:4662-71.

[16] Olivares O, Mayers JR, Gouirand V, Torrence ME, Gicquel T, Borge L, et al. Collagen-derived proline promotes pancreatic ductal adenocarcinoma cell survival under nutrient limited conditions. Nat Commun. 2017;8:16031.

[17] Valle S, Martin-Hijano L, Alcala S, Alonso-Nocelo M, Sainz B, Jr. The Ever-Evolving Concept of the Cancer Stem Cell in Pancreatic Cancer. Cancers (Basel). 2018;10.

[18] Puls TJ, Tan X, Whittington CF, Voytik-Harbin SL. 3D collagen fibrillar microstructure guides pancreatic cancer cell phenotype and serves as a critical design parameter for phenotypic models of EMT. PLoS One. 2017;12:e0188870.

[19] Phillips P. Pancreatic stellate cells and fibrosis. In: Grippo PJ, Munshi HG, editors. Pancreatic Cancer and Tumor Microenvironment. Trivandrum (India)2012. 
[20] Moir JA, Mann J, White SA. The role of pancreatic stellate cells in pancreatic cancer. Surgical oncology. 2015;24:232-8.

[21] von Ahrens D, Bhagat TD, Nagrath D, Maitra A, Verma A. The role of stromal cancer-associated fibroblasts in pancreatic cancer. Journal of hematology \& oncology. 2017;10:76.

[22] McCarroll JA, Naim S, Sharbeen G, Russia N, Lee J, Kavallaris M, et al. Role of pancreatic stellate cells in chemoresistance in pancreatic cancer. Front Physiol. 2014;5:141.

[23] Bolm L, Cigolla S, Wittel UA, Hopt UT, Keck T, Rades D, et al. The Role of Fibroblasts in Pancreatic Cancer: Extracellular Matrix Versus Paracrine Factors. TransI Oncol. 2017;10:578-88.

[24] Shan T, Chen S, Chen X, Lin WR, Li W, Ma J, et al. Cancer-associated fibroblasts enhance pancreatic cancer cell invasion by remodeling the metabolic conversion mechanism. Oncol Rep. 2017;37:1971-9.

[25] Yang XP, Liu SL, Xu JF, Cao SG, Li Y, Zhou YB. Pancreatic stellate cells increase pancreatic cancer cells invasion through the hepatocyte growth factor /c-Met/survivin regulated by P53/P21. Experimental cell research. 2017;357:79-87.

[26] Hwang RF, Moore T, Arumugam T, Ramachandran V, Amos KD, Rivera A, et al. Cancer-associated stromal fibroblasts promote pancreatic tumor progression. Cancer Res. 2008;68:918-26.

[27] Sherman MH, Yu RT, Tseng TW, Sousa CM, Liu S, Truitt ML, et al. Stromal cues regulate the pancreatic cancer epigenome and metabolome. Proc Natl Acad Sci U S A. 2017;114:1129-34.

[28] Dalla Pozza E, Dando I, Biondani G, Brandi J, Costanzo C, Zoratti E, et al. Pancreatic ductal adenocarcinoma cell lines display a plastic ability to bidirectionally convert into cancer stem cells. Int $\mathrm{J}$ Oncol. 2014;46:1099-108.

[29] Deer EL, Gonzalez-Hernandez J, Coursen JD, Shea JE, Ngatia J, Scaife CL, et al. Phenotype and genotype of pancreatic cancer cell lines. Pancreas. 2010;39:425-35.

[30] Kim Y, Han D, Min H, Jin J, Yi EC. Comparative proteomic profiling of pancreatic ductal adenocarcinoma cell lines. Mol Cells. 2014;37:888-98.

[31] Leca J, Martinez S, Lac S, Nigri J, Secq V, Rubis M, et al. Cancer-associated fibroblast-derived annexin A6+ extracellular vesicles support pancreatic cancer aggressiveness. J Clin Invest. 2016;126:4140-56.

[32] Zeeberg K, Cardone RA, Greco MR, Saccomano M, Nohr-Nielsen A, Alves F, et al. Assessment of different 3D culture systems to study tumor phenotype and chemosensitivity in pancreatic ductal adenocarcinoma. Int J Oncol. 2016;49:243-52.

[33] Valenti G, Quinn HM, Heynen G, Lan L, Holland JD, Vogel R, et al. Cancer Stem Cells Regulate CancerAssociated Fibroblasts via Activation of Hedgehog Signaling in Mammary Gland Tumors. Cancer Res. 
2017;77:2134-47.

[34] Su S, Chen J, Yao H, Liu J, Yu S, Lao L, et al. CD10(+)GPR77(+) Cancer-Associated Fibroblasts Promote Cancer Formation and Chemoresistance by Sustaining Cancer Stemness. Cell. 2018;172:84156 e16.

[35] Busco G, Cardone RA, Greco MR, Bellizzi A, Colella M, Antelmi E, et al. NHE1 promotes invadopodial ECM proteolysis through acidification of the peri-invadopodial space. FASEB J. 2010;24:3903-15.

[36] Chu GC, Kimmelman AC, Hezel AF, DePinho RA. Stromal biology of pancreatic cancer. J Cell Biochem. 2007;101:887-907.

[37] Shields MA, Dangi-Garimella S, Krantz SB, Bentrem DJ, Munshi HG. Pancreatic cancer cells respond to type I collagen by inducing snail expression to promote membrane type 1 matrix metalloproteinasedependent collagen invasion. J Biol Chem. 2011;286:10495-504.

[38] Rhim AD, Mirek ET, Aiello NM, Maitra A, Bailey JM, McAllister F, et al. EMT and dissemination precede pancreatic tumor formation. Cell. 2012;148:349-61.

[39] Cardone RA, Greco MR, Zeeberg K, Zaccagnino A, Saccomano M, Bellizzi A, et al. A Novel NHE1Centered Signaling Cassette Drives Epidermal Growth Factor Receptor-Dependent Pancreatic Tumor Metastasis and Is a Target for Combination Therapy. Neoplasia. 2015;17:155-66.

[40] Mostafa ME, Erbarut-Seven I, Pehlivanoglu B, Adsay V. Pathologic classification of "pancreatic cancers": current concepts and challenges. Chinese clinical oncology. 2017;6:59.

[41] Zhao Y, Bao Q, Renner A, Camaj P, Eichhorn M, Ischenko I, et al. Cancer stem cells and angiogenesis. Int J Dev Biol. 2011;55:477-82.

\section{Figures}


A

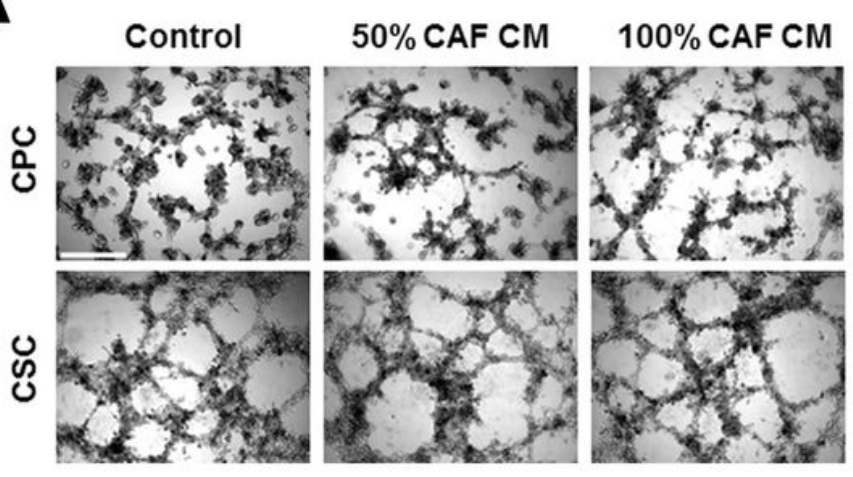

B
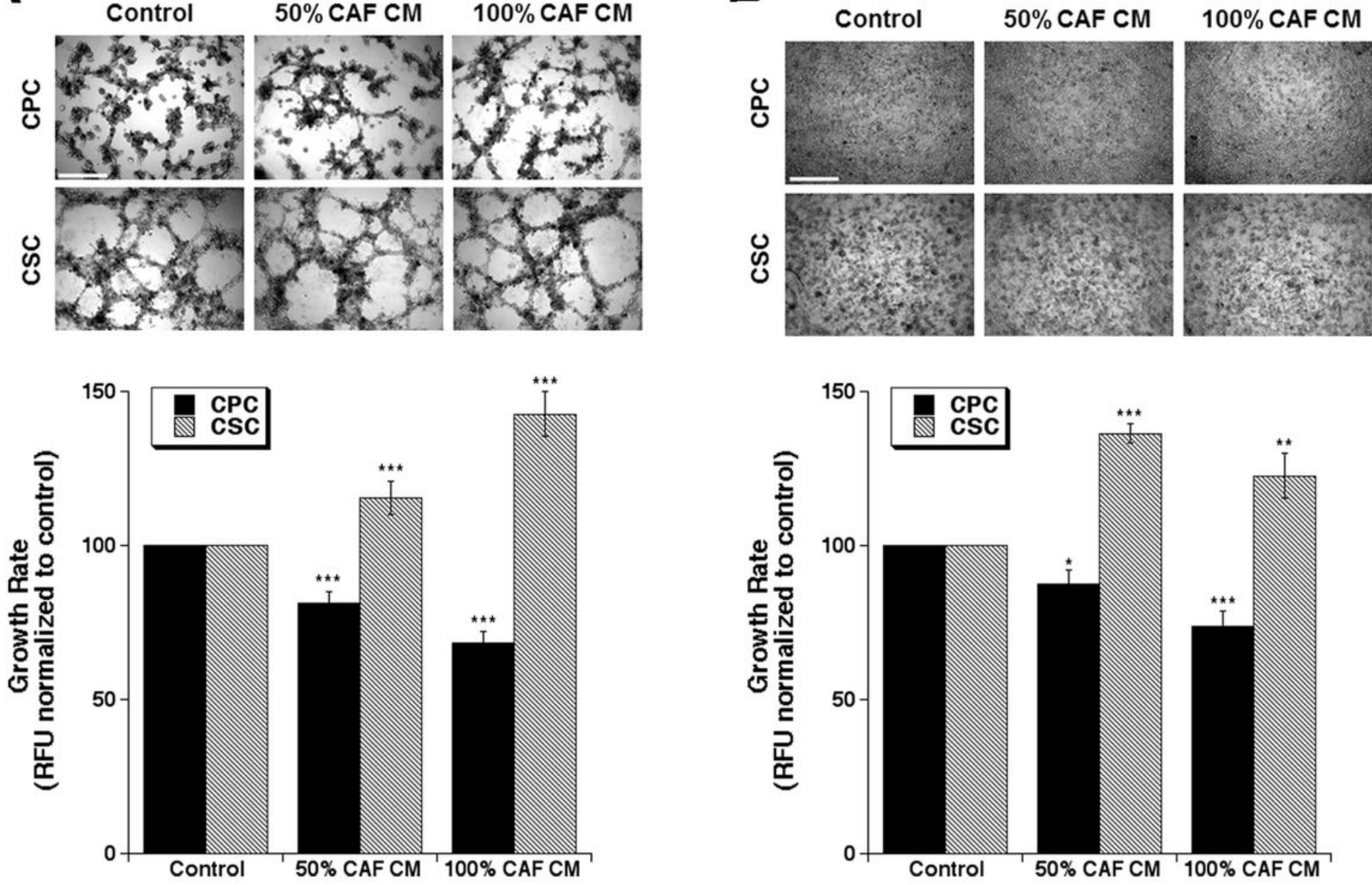

Figure 1

Figure 1. CAF CM inhibits CPC growth and stimulates CSC growth on both ECM compositions. A) Representative microscopic images of growth morphology of Panc1 CPCs and their derived CSCs cultured on organotypic cultures composed of $90 \%$ Matrigel:10\% Collagen I (left panel) and 20\%

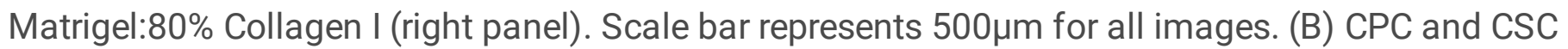
growth rates in organotypic cultures of $90 \%$ Matrigel:10\% Collagen I (left panel) and $20 \%$ Matrigel:80\% Collagen I (right panel). Growth rates of CPCs and CSCs cultured on the different ECMs were calculated from Resazurin reduction assays according to the standard curves obtained by fluorescence readings of Resazurin and are normalized to the control as 100 as described in Materials and Methods. Data are mean \pm SEM, $n=5,{ }^{*} p<0.05,{ }^{*} p<0.01,{ }^{* \star *} p<0.001$ to the control of each cell type on each matrix. 
A
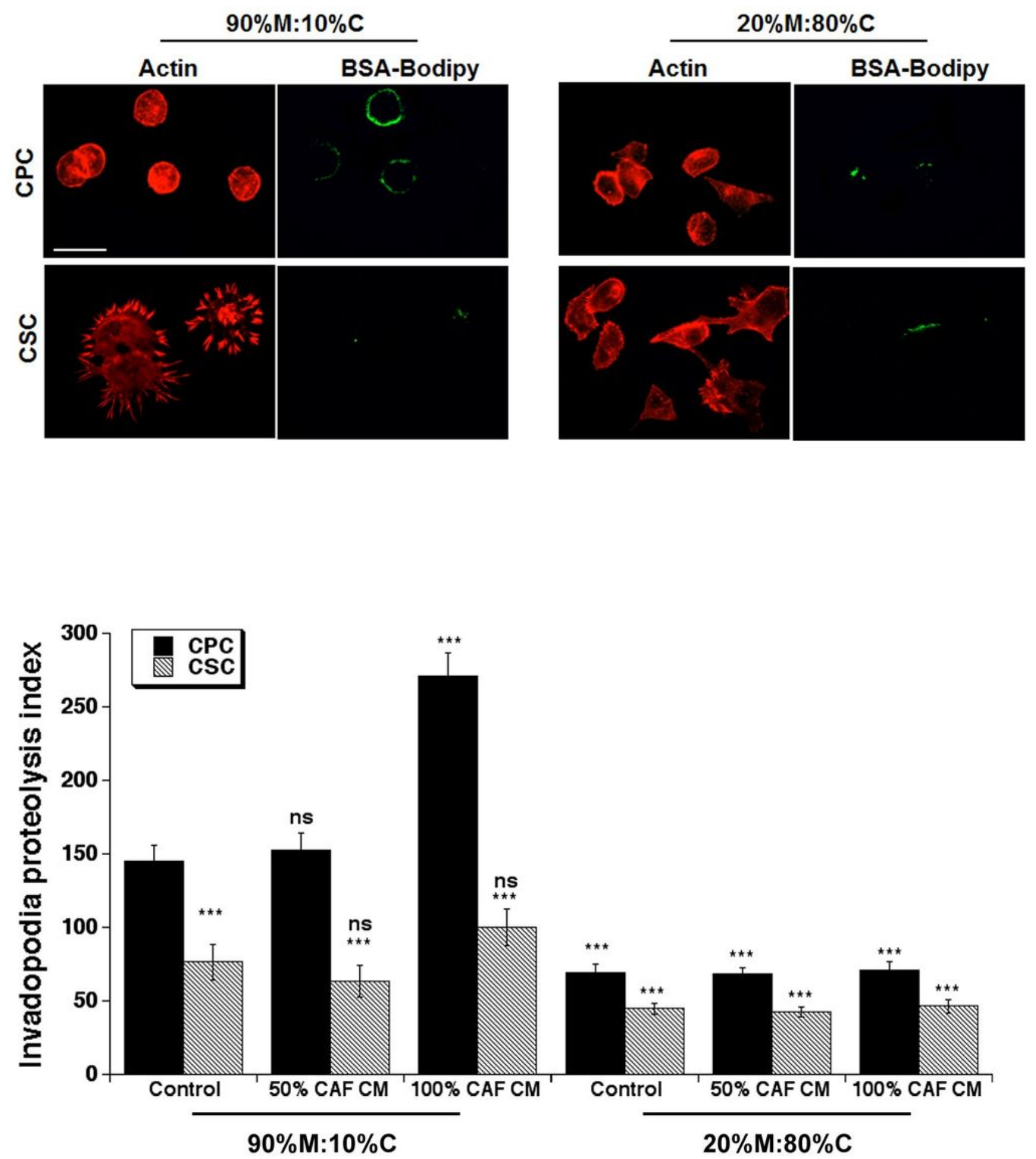

Figure 2

Figure 1. CAF CM inhibits CPC growth and stimulates CSC growth on both ECM compositions. A) Representative microscopic images of growth morphology of Panc1 CPCs and their derived CSCs cultured on organotypic cultures composed of 90\% Matrigel:10\% Collagen I (left panel) and 20\% Matrigel:80\% Collagen I (right panel). Scale bar represents 500 $\mu \mathrm{m}$ for all images. (B) CPC and CSC growth rates in organotypic cultures of 90\% Matrigel:10\% Collagen I (left panel) and 20\% Matrigel:80\% 
Collagen I (right panel). Growth rates of CPCs and CSCs cultured on the different ECMs were calculated from Resazurin reduction assays according to the standard curves obtained by fluorescence readings of Resazurin and are normalized to the control as 100 as described in Materials and Methods. Data are mean $\pm S E M, n=5,{ }^{*} p<0.05,{ }^{* *} p<0.01,{ }^{* *} p<0.001$ to the control of each cell type on each matrix.

A
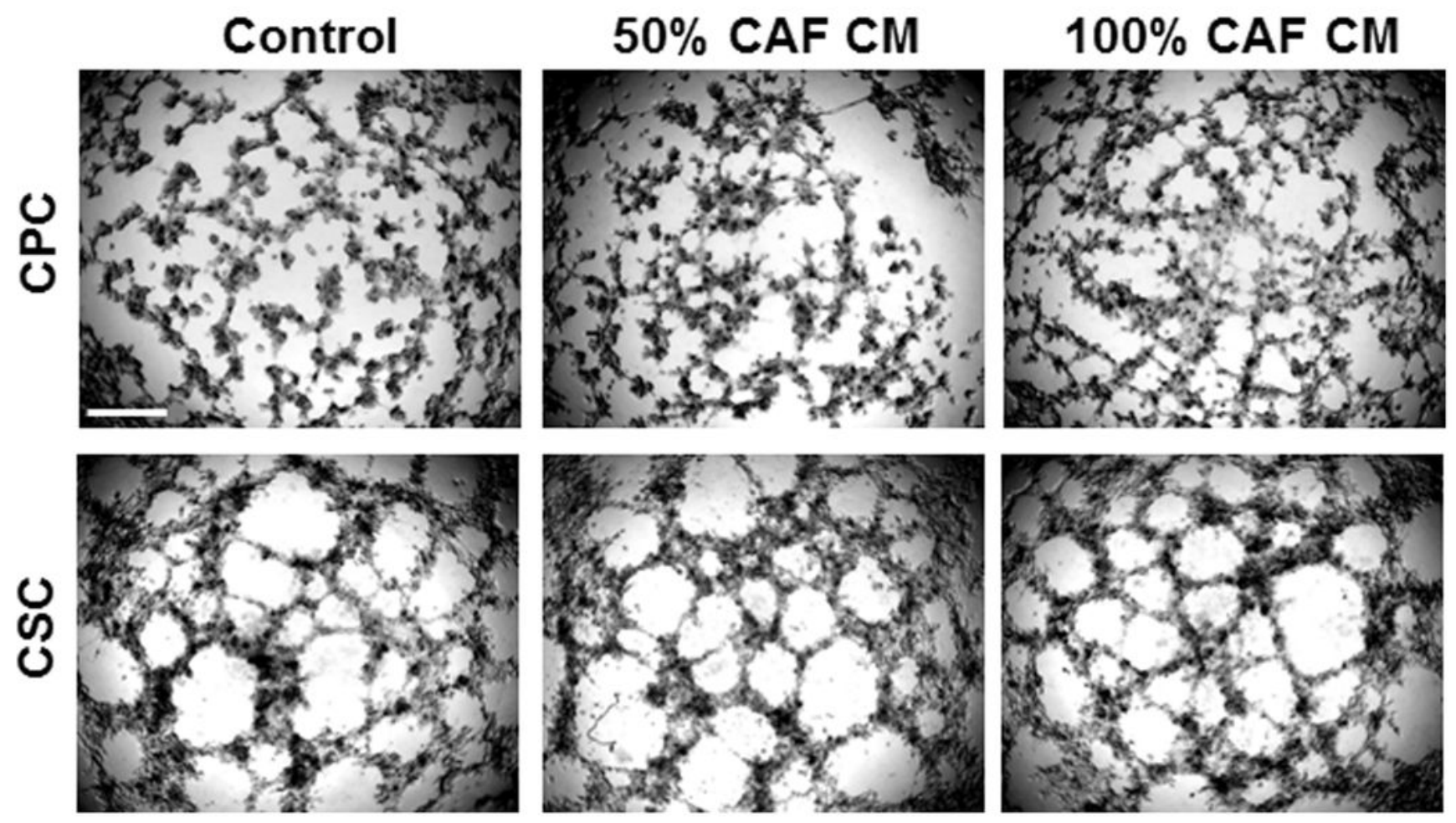

B
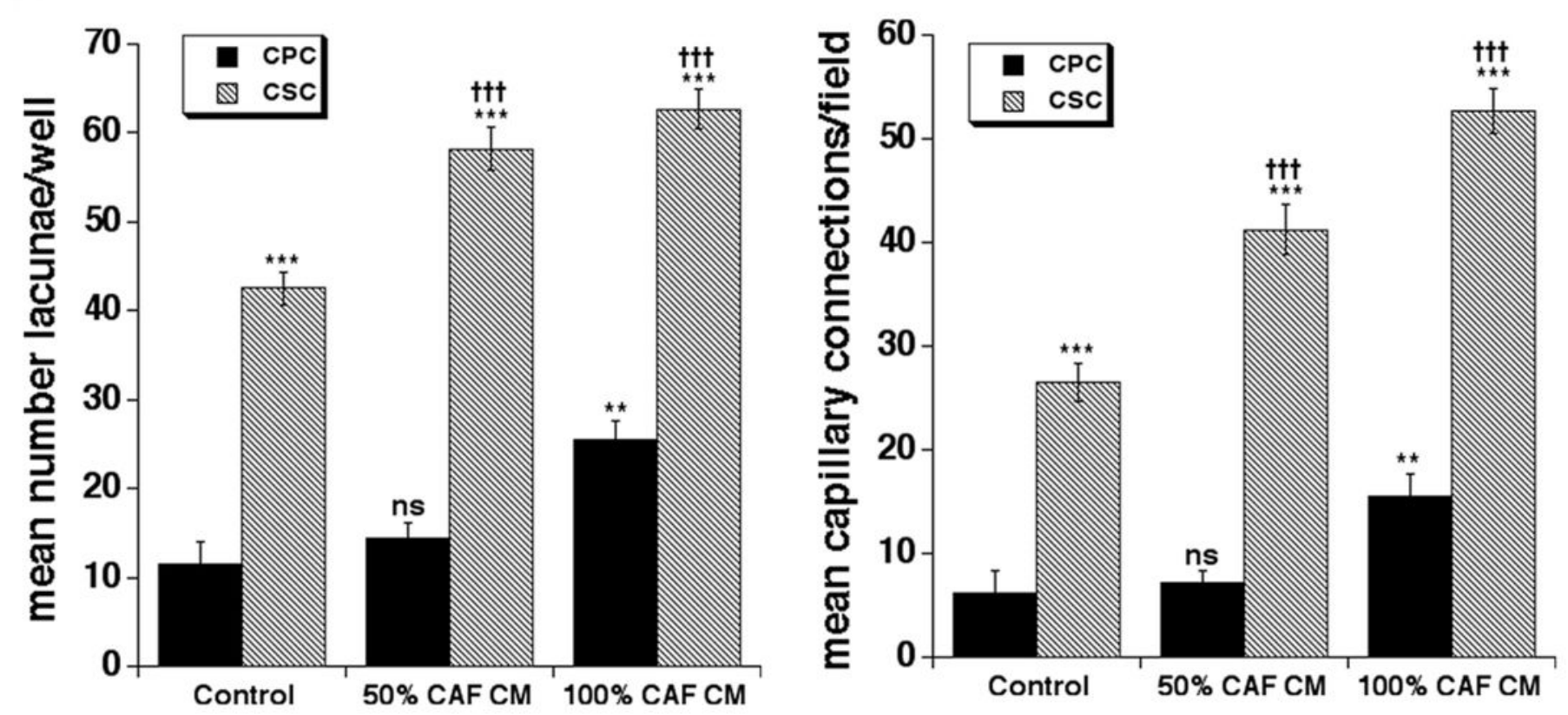

Figure 3 
Figure 3. CAF secretome stimulates the vascular-like morphology (VM) of both CSCs and CPCs grown on $90 \%$ Matrigel: $10 \%$ Collagen I. ECM composition modifies the effect of CAF CM on vasculogenic mimicry in CPCs and CSCs. Cells were grown on 90\% Matrigel:10\% Collagen I and after $24 \mathrm{hrs}$ to permit their adherence the cultures were incubated with CAF CM as described in Materials and Methods. A) Representative microscopic images of growth morphology of CPCs and their derived CSCs cultured on organotypic cultures composed of $90 \%$ Matrigel: $10 \%$ Collagen I for 5 days with their growth medium or with either $50 \%$ or $100 \%$ of the CAF conditioned medium. Scale bar represents $500 \mu \mathrm{m}$ for all images. (B) After 7 days in these growth conditions, vascular channel networks were analysed as described in Material and Methods for mean number of lacunae per well (left panel) and mean number of capillary connections per field (right panel). Mean \pm SEM from three independent experiments, ns, non significant, ${ }^{\star *} p<0.01,{ }^{* \star *} p<0.001$ compared to the CPC control; $+\dagger+p<0.001$ CSCs compared to their control.

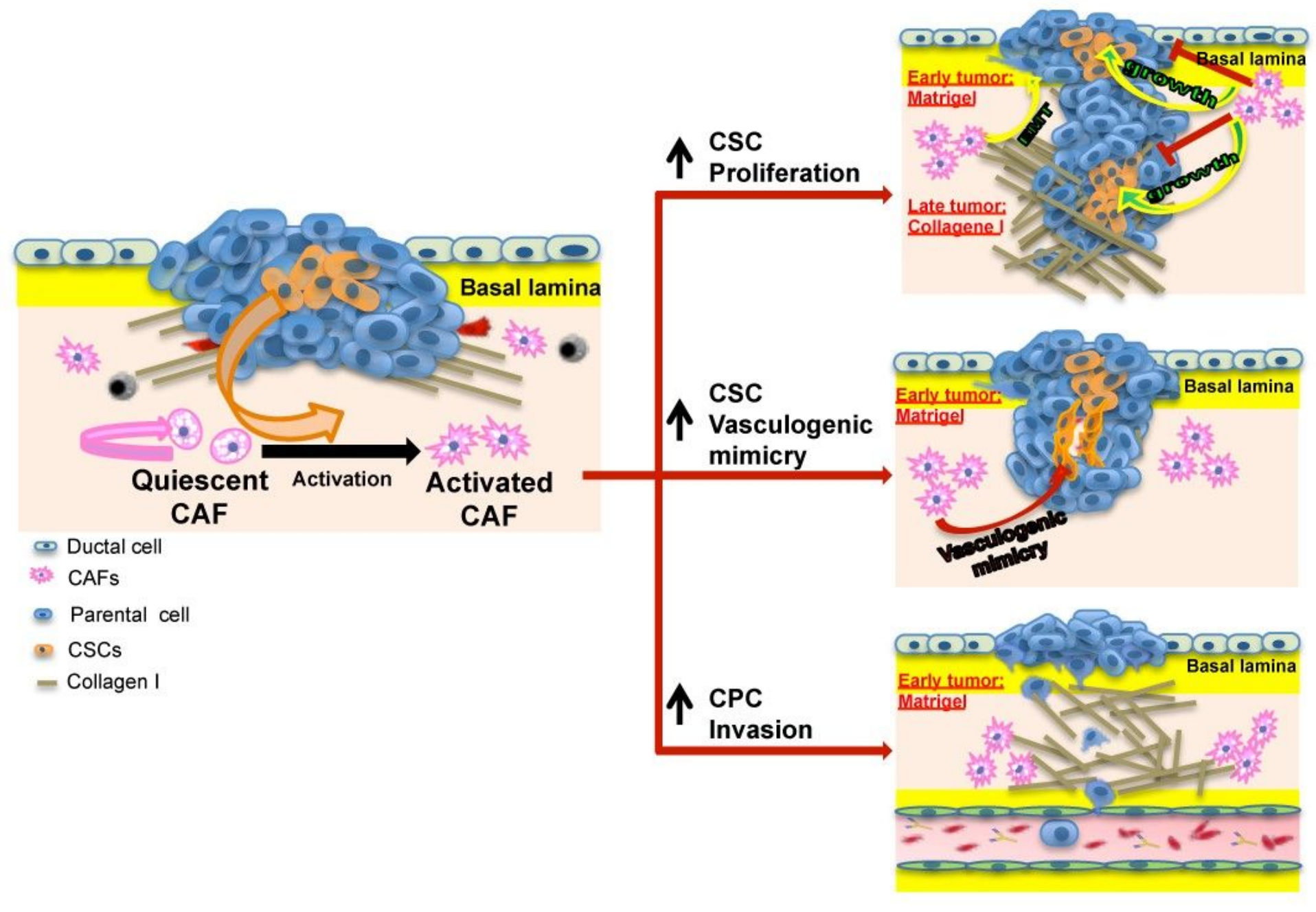

Figure 4

Figure 4. Model of Influence of the ECM composition on the CAF-dependent regulation of CPC and CSC plasticity. Matrigel induces the formation of autocrine loops in CSCs. Indeed, on Matrigel (1) CSCs secrete a high amount of potent proangiogenic and growth factors (ie. PDGF, MMP9, IL8, EGF, HGF, bFGF, ET-1) [5], which, via paracrine mechanisms, stimulate CAF growth, which in turn secrete factors that increase CSC growth/self-renewal and vasculogenic mimicry while decreasing CPC growth but increasing CPC 
invasion. This creates a vicious positive-feedback growth cycle between the CAFs and CSCs to increase the stemness of the tumor while exacerbating the aggressive angiogenesis phenotype of the CSCs and invasive phenotype of the CPCs.

\section{Supplementary Files}

This is a list of supplementary files associated with this preprint. Click to download.

- supplement1.tif 